\title{
Condições de saúde de mulheres e homens idosos com idade avançada: estudo longitudinal
}

\author{
Health status of older men and women: longitudinal study
}

Condiciones de salud de mujeres y hombres de edad avanzada: un estudio longitudinal

\author{
Darlene Mara dos Santos Tavares ' @ , Nayara Gomes Nunes Oliveira' @

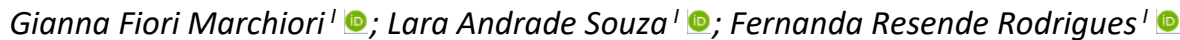

'Universidade Federal do Triângulo Mineiro, Uberaba, MG, Brasil

\begin{abstract}
RESUMO
Objetivo: descrever as características sociodemográficas e de saúde de mulheres e homens com 75 anos ou mais de idade, no baseline e follow-up de quatro anos e verificar para mulheres e homens as mudanças nas condições de saúde. Métodos: estudo longitudinal com 109 idosos de 75 anos ou mais de idade de um município no Triângulo Mineiro. A coleta dos dados, realizada em dois momentos (2014-2018), ocorreu no domicílio com a aplicação de instrumentos validados no Brasil. Procederam-se às análises descritiva e teste $t$ pareado $(p<0,05)$. Os projetos foram aprovados pelo Comitê de Ética e Pesquisa com Seres Humanos. Resultados: verificaram-se, em ambos os sexos, aumento do número de morbidades e diminuição do escore total das atividades instrumentais da vida diária. Entre as mulheres observou-se, ainda, aumento do número de quedas e do escore de fragilidade. Conclusão: ao longo do seguimento houve piora nas condições de saúde dos idosos, sendo mais expressiva entre as mulheres. Descritores: Estudos longitudinais; Enfermagem Geriátrica; Idoso; Idoso de 80 Anos ou mais; Nível de Saúde.
\end{abstract}

\begin{abstract}
Objective: to describe the sociodemographic and health characteristics of women and men aged 75 or over, at baseline and after four years of follow-up, and to ascertain changes in their health status. Methods: in this longitudinal study of 109 elderly people aged 75 or over from a city in the Triângulo Mineiro, data were collected at two points (2014 and 2018), at home, by applying instruments validated for use in Brazil. Descriptive analysis and paired t-tests were performed $(p<0.05)$. The projects were approved by the human research ethics committee. Results: in both genders, the number of morbidities increased and the total score for instrumental activities of daily living decreased. Among women, the number of falls and frailty score also increased. Conclusion: the older people's health status worsened over the course of follow-up, more so among the women. Descriptors: Longitudinal Studies; Geriatric Nursing; Aged; Aged, 80 and over; Health Status.
\end{abstract}

\section{RESUMEN}

Objetivo: describir las características sociodemográficas y de salud de mujeres y hombres de 75 años o más, en la base de referencia y el seguimiento durante cuatro años, y verificar los cambios en las condiciones de salud de mujeres y hombres. Métodos: estudio longitudinal con 109 personas mayores, de 75 años o más, de un municipio del Triângulo Mineiro. La recolección de datos, realizada en dos momentos (2014-2018), se realizó en sus domicilios aplicando instrumentos validados en Brasil. Se realizaron análisis descriptivos y prueba t pareada $(p<0.05)$. Los proyectos fueron aprobados por el Comité de Ética en Investigación con Humanos. Resultados: en ambos os sexos, hubo un aumento en el número de morbilidades y una disminución en la puntuación total de las actividades instrumentales de la vida diaria. Entre las mujeres, se observó asimismo un aumento en el número de caídas y la puntuación de fragilidad. Conclusión: a lo largo del seguimiento, las condiciones de salud de las personas mayores empeoraron, más expresivamente entre las mujeres.

Descriptores: Estudios Longitudinales; Enfermería Geriátrica; Anciano; Anciano de 80 o más Años; Estado de Salud.

\section{INTRODUÇÃO}

O envelhecimento populacional é uma tendência mundial, com maior crescimento entre os grupos etários com idade mais avançada. No Brasil, os idosos com 75 anos ou mais de idade representam 3,8\% da população e estima-se que em 2060 esse percentual seja de 13,4\%. A partir de 2000 a expectativa de vida dos brasileiros aumentou de forma acentuada, contribuindo para um acréscimo no número da população idosa. Em 2000 a expectativa de vida era de 69,8 anos; em 2010 de 73,9 anos e, atualmente 76,7 anos, sendo maior para as mulheres $(80,2$ anos) em relação aos homens $(73,2 \text { anos })^{1}$.

Como observado na expectativa de vida, o envelhecimento populacional tem ocorrido com a feminização, ou seja, há maior proporção de pessoas do sexo feminino na população idosa, especialmente em idades mais avançadas. Entretanto, entre as mulheres, a maior probabilidade de trabalhar no setor informal, bem como níveis mais baixos de escolaridade e renda, e o maior número de morbidades e incapacidades funcionais, estão entre os principais fatores

Agradecimentos ao Conselho Nacional de Desenvolvimento Científico e Tecnológico (CNPq), processo número 407978/2016-0, e à Fundação de Amparo à Pesquisa do Estado de Minas Gerais (FAPEMIG), processo número APQ - 0189417

Autora correspondente: Darlene Mara dos Santos Tavares. E-mail: darlene.tavares@uftm.edu.br

Editora Científica: Cristiane Helena Gallasch; Editora Associada: Thelma Spindola 
que contribuem para uma menor proteção, segurança e bem-estar na velhice. No que se refere aos homens, uma das preocupações é a necessidade de promoção do autocuidado, além da limitação da rede de apoio social devido ao afastamento do trabalho pela aposentadoria, os tornando mais vulneráveis socialmente e nos cuidados com a saúde ${ }^{2}$.

Frente às diferenças entre mulheres e homens idosos em seus papéis e oportunidades ao longo da vida ${ }^{1,2}$, uma abordagem segundo o sexo é essencial no planejamento de políticas públicas. Além disto, a atenção à saúde do idoso, com idade mais avançada, é premente considerando o seu crescimento no Brasil. Nesta perspectiva, reforça-se a necessidade do desenvolvimento de estudos longitudinais, delineados de maneira a permitir inferências causais, além de acompanhar a evolução da condição de saúde segundo sexo de idosos com 75 anos ou mais de idade.

Contudo, apesar da relevância, destaca-se a escassez de investigações longitudinais que analisaram as mudanças nas condições de saúde segundo sexo de idosos com idade mais avançada. A produção científica se concentra em estudos com delineamento transversal ${ }^{2-6}$, que são importantes para a prática clínica, mas não possibilitam a análise de causalidade. Já as pesquisas longitudinais, a maioria foi desenvolvida entre idosos com 60 anos ou mais de idade ${ }^{7-13}$; e abordaram condições específicas de saúde, como fragilidade ${ }^{7,8}$, quedas ${ }^{12,13}$, capacidade funcional e desempenho físico $^{11}$, bem como as realizadas com idosos longevos ${ }^{14-17}$. Além disto, os dados não foram analisados e descritos de acordo com o sexo.

Assim, ao considerar o aumento da população idosa com idade mais avançada ${ }^{1}$, a relevância da abordagem segundo o sexo para o desenvolvimento de ações de saúde ${ }^{1,2}$ e a escassez de estudos longitudinais com essa temática, o objetivo desse estudo foi descrever as características sociodemográficas e de saúde de mulheres e homens com 75 anos ou mais de idade, no baseline e follow-up de quatro anos e verificar para mulheres e homens as mudanças nas condições de saúde.

\section{MÉTODO}

Estudo de abordagem quantitativa, tipo inquérito domiciliar, longitudinal, desenvolvido na área urbana de um município do Triângulo Mineiro, Minas Gerais (MG).

Os dados foram coletados por meio da entrevista direta, no domicílio dos idosos, em dois momentos: baseline março a julho de 2014 - e follow-up de quatro anos - março a julho de 2018.

A amostra foi constituída por idosos com 75 anos ou mais de idade, entrevistados na pesquisa "Dependência para as atividades da vida diária, fragilidade e uso de serviços de saúde entre idosos do Triângulo Mineiro". No referido estudo utilizou-se amostragem por conglomerado em múltiplo estágio e excluíram-se idosos institucionalizados; com problemas de comunicação como surdez, não corrigida por aparelhos, transtornos graves da fala e com declínio cognitivo.

$\mathrm{Na}$ atual investigação foram incluídos idosos com 75 anos ou mais de idade, que residiam na área urbana de um município no Triângulo Mineiro (MG) e foram entrevistados em 2014 e 2018. No banco de dados, verificou que 109 idosos atendiam aos critérios estabelecidos, sendo 79 mulheres e 30 homens.

Em ambos os momentos, os entrevistadores selecionados com experiência prévia, receberam treinamento quanto ao preenchimento dos instrumentos de coleta dos dados, forma de abordar o idoso e questões éticas na condução de pesquisa.

O declínio cognitivo, um dos critérios de exclusão, foi avaliado pelo Miniexame do Estado Mental. Consideraram-se os pontos de corte: $\leq 13$ para analfabetos, $\leq 18$ para escolaridade média ( $1-12$ anos) e $\leq 26$ para alta escolaridade $(\geq 12 \text { anos })^{18}$.

Os dados sociodemográficos, morbidades, ocorrência de quedas e a autoavaliação da saúde foram obtidos mediante a aplicação do questionário estruturado elaborado pelos membros do Grupo de Pesquisa em Saúde Coletiva.

A capacidade funcional nas atividades instrumentais da vida diária (AIVD) foi mensurada pela Escala de Atividades Instrumentais da Vida Diária, adaptada no Brasil. A classificação das AIVD varia de sete (maior nível de dependência) a 21 pontos (independência completa), sendo o idoso classificado como: dependente total (7 pontos), parcial (8 a 20 pontos) e independente (21 pontos) ${ }^{19}$

A síndrome de fragilidade foi identificada por meio dos cinco componentes do fenótipo de fragilidade ${ }^{20}:$ (1) perda de peso não intencional; (2) diminuição da força muscular; (3) lentidão na velocidade de marcha; (5) baixa nível atividade física; e (5) autorrelato de exaustão e/ou fadiga, conforme descrito em estudo prévio ${ }^{21}$. Com base nessa avaliação, os idosos com comprometimento em três ou mais desses itens foram classificados como frágeis; enquanto aqueles com um ou dois como pré-frágeis. Idosos com ausência de comprometimento nos cinco componentes foram considerados não frágeis ${ }^{20}$.

Para mensuração do peso, foi utilizada balança eletrônica digital portátil, tipo plataforma, com capacidade para $150 \mathrm{~kg}$ e precisão de $100 \mathrm{~g}$, com o idoso descalço e usando roupas leves. A estatura $(\mathrm{m})$ foi aferida usando fita métrica flexível e inelástica, com extensão de 1,5 metros, dividida em centímetros e subdivida em milímetros, fixada na parede em um local plano e regular, sem rodapé. Aferiu-se com o idoso descalço, em posição ortostática com os pés unidos, de costas para o 
marcador, com o olhar no horizonte. O Índice de Massa Corporal (IMC) foi calculado em $\mathrm{kg} / \mathrm{m}^{2}$ e para sua classificação utilizaram os pontos de corte: baixo peso $\left(\leq 22 \mathrm{~kg} / \mathrm{m}^{2}\right)$, eutrofia $\left(22\right.$ a $\left.27 \mathrm{~kg} / \mathrm{m}^{2}\right)$ e sobrepeso $\left(\geq 27 \mathrm{~kg} / \mathrm{m}^{2}\right)^{22}$.

Para avaliação do desempenho físico utilizou-se a versão brasileira Short Physical Performance Battery (SPPB) composta pela somatória da pontuação adquirida nos testes de equilíbrio, velocidade da marcha e levantar-se da cadeira cinco vezes consecutivas, com escore total que varia de 0 (incapacidade) a 12 (melhor desempenho) pontos. Idosos que obtiveram de 0 a 3 pontos, foram classificados com incapacidade; 4 a 6 pontos, baixo desempenho; 7 a 9 pontos, moderado desempenho e 10 a 12 pontos, bom desempenho ${ }^{23}$

As variáveis estudadas foram as sociodemográficas: sexo (feminino e masculino); arranjo de moradia (só; acompanhado) e renda mensal individual, em salários mínimos (sem rendimento; $\leq 1$; $>1$ ); e as condições de saúde: morbidades (nenhuma; 1|-5; 5 ou mais); número de morbidades (média do número de morbidades); autoavaliação da saúde (negativa; positiva); capacidade funcional nas AIVD (dependente total/parcial e independente); escore de AIVD (média do escore de AIVD); síndrome de fragilidade (não frágil; pré-frágil; frágil); escore de fragilidade (média do número de componentes comprometidos do fenótipo de fragilidade); IMC (baixo peso; eutrófico; sobrepeso); ocorrência de quedas (sim e não); número de quedas (média do número de quedas); desempenho físico (muito ruim/baixo; moderado/bom); e escore de desempenho físico (média do escore de desempenho físico no SPPB).

Construiu-se um banco de dados eletrônico, no programa Excel ${ }^{\circledR}$, com dupla digitação. Após a verificação das inconsistências entre as duas bases de dados e sua correção, o banco de dados foi importado para o programa "Statistical Package for the Social Sciences" (SPSS ${ }^{\circledR}$ ) versão 22.0, para análise.

Os dados foram submetidos às análises de frequências absoluta e relativa. Para verificar as mudanças nas condições de saúde de mulheres e homens com 75 anos ou mais de idade, no baseline e follow-up de quatro anos, foi utilizado o teste $t$ pareado $(p<0,05)$. Para tal análise, as variáveis: morbidades; capacidade funcional para as AIVD; desempenho físico; quedas e fragilidade foram utilizadas na forma quantitativa.

Os projetos foram aprovados pelo Comitê de Ética e Pesquisa com Seres Humanos. As entrevistas foram realizadas após a anuência dos participantes e assinatura do termo de consentimento livre e esclarecido.

\section{RESULTADOS}

As características sociodemográficas e de saúde no baseline e follow-up após quatro anos são descrita na Tabela 1.

TABELA 1: Características sociodemográficas e de saúde segundo sexo dos idosos com 75 anos ou mais de idade no baseline e follow-up após quatro anos, da comunidade de um município no Triângulo Mineiro, Minas Gerais, Brasil, 2020.

\begin{tabular}{|c|c|c|c|c|c|c|c|c|c|}
\hline \multirow{3}{*}{ Variáveis } & & \multicolumn{4}{|c|}{ Sexo Feminino } & \multicolumn{4}{|c|}{ Sexo Masculino } \\
\hline & & \multicolumn{2}{|c|}{ Baseline } & \multicolumn{2}{|c|}{ Follow-up } & \multicolumn{2}{|c|}{ Baseline } & \multicolumn{2}{|c|}{ Follow-up } \\
\hline & & $\mathbf{n}$ & $\%$ & $\mathbf{n}$ & $\%$ & 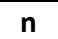 & $\%$ & 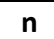 & $\%$ \\
\hline \multirow[t]{2}{*}{ Arranjo Moradia } & Acompanhado (a) & 57 & 72,2 & 65 & 82,3 & 22 & 73,3 & 24 & 80,0 \\
\hline & Sozinho (a) & 22 & 27,8 & 14 & 17,7 & 8 & 26,7 & 6 & 20,0 \\
\hline \multirow[t]{3}{*}{ Renda Individual } & Sem rendimentos & 6 & 7,6 & 4 & 5,1 & 1 & 3,3 & 0 & 0,0 \\
\hline & $\leq 1$ & 46 & 58,2 & 45 & 57,0 & 15 & 50,0 & 12 & 40,0 \\
\hline & $>1$ & 27 & 34,2 & 30 & 38,0 & 14 & 46,7 & 18 & 60,0 \\
\hline \multirow[t]{2}{*}{ Atividades Instrumentais da Vida Diária } & Independentes & 18 & 22,8 & 5 & 6,3 & 9 & 30,0 & 4 & 13,3 \\
\hline & Dependentes & 61 & 77,2 & 74 & 93,7 & 21 & 70,0 & 26 & 86,7 \\
\hline \multirow[t]{3}{*}{ Número de morbidades } & Nenhuma & 3 & 3,8 & 0 & 0,0 & 3 & 10,0 & 0 & 0,0 \\
\hline & 1 -5 & 27 & 34,2 & 27 & 34,2 & 13 & 43,3 & 13 & 43,3 \\
\hline & 5 ou mais & 49 & 62,0 & 52 & 65,8 & 14 & 46,7 & 17 & 56,7 \\
\hline \multirow[t]{2}{*}{ Autoavaliação da saúde } & Positiva & 28 & 35,4 & 31 & 39,2 & 17 & 56,7 & 12 & 40,0 \\
\hline & Negativa & 51 & 64,6 & 48 & 60,8 & 13 & 43,3 & 18 & 60,0 \\
\hline \multirow[t]{3}{*}{ Síndrome de Fragilidade } & Não Frágil & 12 & 15,2 & 7 & 8,9 & 1 & 3,3 & 5 & 16,7 \\
\hline & Pré-Frágil & 47 & 59,5 & 32 & 40,5 & 20 & 66,7 & 17 & 56,7 \\
\hline & Frágil & 20 & 25,3 & 40 & 50,6 & 9 & 30,0 & 8 & 26,7 \\
\hline \multirow[t]{3}{*}{ Índice de Massa Corporal } & Eutrófico & 30 & 38,0 & 34 & 43,0 & 16 & 53,3 & 17 & 56,7 \\
\hline & Baixo peso & 14 & 17,7 & 20 & 25,3 & 3 & 10,0 & 6 & 20,0 \\
\hline & Sobrepeso & 35 & 44,3 & 25 & 31,6 & 11 & 36,7 & 7 & 23,3 \\
\hline \multirow[t]{2}{*}{ Ocorrência de Quedas } & Não & 51 & 64,6 & 53 & 66,7 & 20 & 66,7 & 19 & 63,4 \\
\hline & Sim & 28 & 35,4 & 26 & 33,3 & 10 & 33,3 & 11 & 36,6 \\
\hline \multirow[t]{2}{*}{ Desempenho Físico } & Moderado/Bom & 46 & 58,2 & 42 & 53,2 & 27 & 90,0 & 22 & 73,3 \\
\hline & Incapacidade/Baixo & 33 & 41,8 & 37 & 46,8 & 3 & 10,0 & 8 & 26,7 \\
\hline
\end{tabular}

Notas: baseline: 2014; follow-up: seguimento após quatro anos (2018) 
As idosas representaram $72,5 \%$ da amostra. Ao longo do seguimento, verificou-se aumento percentual de mulheres que moravam acompanhadas; da dependência para as AIVD, da presença de 5 ou mais morbidades e da não ocorrência de quedas. Embora tenha diminuído o percentual de idosas que caíram, observou-se aumento do número de quedas recorrentes $(2,03 \pm 1,82)$. Em contrapartida, ocorreu diminuição do percentual de idosas que recebiam $\leq 1$ salário mínimo; que tinham autopercepção de saúde negativa e com desempenho físico moderado/bom. No baseline, maior percentual de idosas foi classificado como pré-frágil, mudando esta condição para frágil no follow-up. O mesmo se observou com o IMC, em que a maioria tinha, inicialmente, sobrepeso e passou para eutrófico.

Entre os homens idosos com 75 anos ou mais de idade, identificou-se, ao longo de quatro anos, aumento do percentual daqueles que moravam acompanhados; dependentes para as AIVD; com 5 ou mais morbidades e eutrófico. Contudo, ocorreu diminuição nas porcentagens das variáveis pré-fragilidade, destacando seu aumento entre os não frágeis; desempenho físico moderado/bom; e não ocorrência de quedas, com aumento de episódios recorrentes $(2,00 \pm 1,33)$. Observou-se, ainda, aumento na renda individual mensal que passou no baseline de $\leq 1$ para >1 salário mínimo no follow-up e na autoavaliação da saúde, em que era positiva na primeira onda, e ficou negativa na segunda.

Quanto às condições de saúde, as Tabela 2 e 3 apresentam dados de comparação das médias das variáveis no baseline e follow-up para mulheres e homens.

TABELA 2: Comparação das médias das variáveis relacionadas às condições de saúde no baseline e follow-up de quatro anos entre mulheres idosas com 75 anos ou mais de idade da comunidade de um município do Triângulo Mineiro, Minas Gerais, Brasil, 2020.

\begin{tabular}{lccc}
\hline \multirow{2}{*}{ Variáveis } & \multicolumn{3}{c}{ Sexo Feminino } \\
\cline { 2 - 4 } & Média & Desvio Padrão & $\boldsymbol{p}^{*}$ \\
\hline Número de morbidades & 6,06 & 3,69 & $\mathbf{0 , 0 2 0}$ \\
$\begin{array}{l}\text { Baseline } \\
\text { Follow-up }\end{array}$ & 7,01 & 3,56 & \\
Escore de AIVD & & & \\
Baseline & 18,05 & 2,83 & $<0,001$ \\
Follow-up & 16,08 & 3,59 & \\
Escore Desempenho Físico & & & \\
$\begin{array}{l}\text { Baseline } \\
\text { Follow-up }\end{array}$ & 6,78 & 2,58 & 0,158 \\
Número de quedas & 6,27 & 3,46 & \\
Baseline & & & \\
Follow-up & 0,64 & 1,01 & 0,003 \\
Escore de Fragilidade & 2,03 & 1,82 & \\
Baseline & & & $<0,001$ \\
Follow-up & 1,68 & 1,13 & \\
\hline Notas: AlVD: Atvidas & 2,43 & 1,32 & \\
\hline
\end{tabular}

Notas: AIVD: Atividades instrumentais de vida diária; Baseline: 2014; Followup: seguimento após quatro anos (2018); Teste $t$ pareado; ${ }^{*} p<0,05$.

Entre as mulheres idosas, com 75 anos ou mais de idade, verificou aumento na média do número de morbidades $(p=0,020)$, do número de quedas $(p=0,003)$ e do escore de fragilidade $(p<0,001)$ ao longo do seguimento. Já a média do escore total de AIVD $(p<0,001)$ foi menor no follow-up, denotando maior dependência nas AIVD.

Observou-se, ao longo do seguimento, aumento na média do número de morbidades $(p=0,018)$ e diminuição do escore de AIVD $(p=0,012)$ entre os homens idosos com 75 anos ou mais de idade. 
TABELA 3: Comparação das médias das variáveis relacionadas às condições de saúde no baseline e follow-up de quatro anos entre homens idosos com 75 anos ou mais de idade da comunidade de um município no Triângulo Mineiro, Minas Gerais, Brasil, 2020.

\begin{tabular}{lccc}
\hline & \multicolumn{3}{c}{ Sexo Masculino } \\
\cline { 2 - 4 } Variáveis & Média & Desvio Padrão & $\boldsymbol{p}^{*}$ \\
\hline $\begin{array}{l}\text { Número de morbidades } \\
\text { Baseline }\end{array}$ & 4,53 & 3,53 & $\mathbf{0 , 0 1 8}$ \\
$\begin{array}{l}\text { Follow-up } \\
\text { Escore de AIVD }\end{array}$ & 5,83 & 2,92 & \\
Baseline & & & \\
Follow-up & 17,73 & 3,06 & $\mathbf{0 , 0 1 2}$ \\
$\begin{array}{l}\text { Escore Desempenho Físico } \\
\text { Baseline }\end{array}$ & 15,76 & 3,72 & \\
$\begin{array}{l}\text { Follow-up } \\
\text { Número de quedas }\end{array}$ & 8,60 & 2,02 & 0,095 \\
$\begin{array}{l}\text { Baseline } \\
\text { Follow-up }\end{array}$ & 7,70 & 3,34 & \\
$\begin{array}{l}\text { Escore de Fragilidade } \\
\text { Baseline }\end{array}$ & 0,80 & 1,09 & 0,066 \\
Follow-up & 2,00 & 1,33 & \\
\hline
\end{tabular}

Notas: AIVD: Atividades instrumentais de vida diária; Baseline: 2014; Follow-up: seguimento após quatro anos (2018); Teste $t$ pareado; ${ }^{*} p<0,05$

\section{DISCUSSÃO}

O maior percentual de idosos com 75 anos ou mais de idade, em ambos os sexos, que morava acompanhado, está em consonância com estudos nacionais desenvolvidos na comunidade ${ }^{3,6,24}$. Estes achados trazem a reflexão de quais fatores levaram os idosos, que residiam em domicílio unipessoal, passar a compartilhar a moradia ao longo do tempo. Um dos aspectos pode estar relacionado às possíveis limitações funcionais, mais frequentes com o avançar da idade, e a consequente necessidade de auxílio para realização das atividades cotidianas e acesso aos serviços de saúde. Assim, o apoio social, em especial dos familiares, torna-se essencial|3,6,24.

Ao longo do seguimento observou diminuição percentual da renda $\leq 1$ salário mínimo em ambos os grupos, sendo o aumento da renda maior entre aqueles do sexo masculino. Esse fato pode estar relacionado à manutenção da participação dos idosos com 75 anos ou mais de idade no mercado de trabalho, além da possibilidade de exercerem atividades laborais autônomas para aumentar os rendimentos ${ }^{25}$, que nesta etapa da vida pode não ser tão benéfica. 0 percentual superior de homens idosos com maior renda pode ser compreendido pela situação das mulheres, ao longo da vida, que mantêm papel de cuidadoras dos familiares, juntamente com a realização das tarefas domésticas, dedicando-se menos ao mercado de trabalho formal, em comparação aos homens ${ }^{2,26}$.

O aumento da dependência para realizar as AIVD corrobora estudos longitudinais entre idosos no geral de ambos os $\operatorname{sexos}^{9,27}$. Com o avançar da idade, podem ocorrer alterações biológicas, dentre elas, sarcopenia, diminuição da força muscular e da velocidade de marcha e instabilidade postural ${ }^{24}$, impactando negativamente na realização das atividades cotidianas. Ademais, o declínio funcional inicia-se pelas atividades que exigem maior coordenação e habilidades, como as AIVD ${ }^{27}$.

Assim como neste estudo, em investigação longitudinal conduzida com idosos verificou-se aumento de doenças crônicas no seguimento de quatro anos ${ }^{8}$. Com o avançar da idade, as doenças crônicas tornam-se mais frequentes, em razão de alterações fisiológicas que impactam na saúde física dos longevos ${ }^{4}$. Na atual pesquisa, o percentual de idosos que apresentaram cinco ou mais morbidades, no follow-up, foi maior entre os homens. Assim, torna-se necessário propor ações em saúde que evolvam as particularidades dos idosos homens e mulheres, com 75 anos ou mais de idade, além de se considerar os aspectos culturais sobre os comportamentos masculino e feminino no autocuidado ${ }^{2}$.

Em ambos os momentos, as mulheres referiram autoavaliação da saúde negativa semelhante ao estudo com idosos no sul do Brasil ${ }^{28}$. A maior expectativa de vida das mulheres pode ocorrer com o aumento de doenças crônicas e declínio funcional que, por sua vez, influenciam negativamente na autoavaliação da saúde ${ }^{26}$. Entre os homens idosos com 75 anos ou mais de idade, após o follow-up, houve mudança na autoavaliação da saúde postiva para negativa. Em estudo internacional identificou-se que $26,9 \%$ dos homens na faixa etária de $80-90$ anos relataram autoperceção negativa de saúde ${ }^{29}$. A maior propensão dos efeitos cumulativos, ocasionados pelo baixo nível econômico; falta de 
suporte social ${ }^{26}$; alterações físicas, emocionais e sociais podem impactar na autoavaliação de saúde ${ }^{14}$. Assim, fatores multidimensionais interferem na autoavaliação da saúde, o que requer ações multidisciplinares com ênfase na promoção da saúde e na prevenção de agravos.

Observou aumento percentual de mulheres idosas com 75 anos e mais de idade, no follow-up, que se tornaram frágeis, condizente com pesquisas longitudinais ${ }^{7,14}$. A maior expectativa de vida das mulheres associada às alterações endócrinas decorrentes da menopausa, que causa a queda dos níveis hormonais, redução da massa e força muscular resulta na maior vulnerabilidade à fragilidade neste grupo ${ }^{3}$. Ainda que a oxidação celular; as modificações neuromusculares, a desregulação do sistema neuroendócrino e a disfunção do sistema imunológico tornam os idosos mais vulneráveis à fragilidade ${ }^{30}$, entre os homens, ao longo do seguimento, verificou-se diminuição percentual de frágeis e pré-frágeis e aumento dos não frágeis. Considerando os eventos adversos à saúde relacionados à fragilidade ${ }^{7,14}$, é essencial que o enfermeiro da estratégia da saúde da família faça a avaliação dessa condição, com intuito de postergar seus efeitos.

Em estudo longitudinal com follow-up de oito anos entre idosos coreanos, com 65 anos e mais de idade, identificou-se que $52,9 \%$ das mulheres eram obesas ${ }^{31}$, divergindo dos achados desta pesquisa em que, ao término do seguimento, a maioria era eutrófica. As alterações dos mecanismos fisiológicos que regulam a saciedade, com o avançar da idade, associados ao estilo de vida, condições de saúde, fatores sociais e ambientais podem afetar diretamente a perda de apetite e/ou diminuição da ingestão alimentar entre idosos ${ }^{32}$. Estes resultados são positivos, tendo em vista que o sobrepeso pode estar relacionado ao surgimento de doenças crônicas ${ }^{32}$, e que entre as idosas, desta pesquisa, prevaleceram aquelas com polimorbidade. Entre os homens, nos dois momentos, predominaram os eutrófico corroborando investigação realizada na comunidade entre longevos ${ }^{33}$ e divergindo de pesquisa internacional, na qual $48 \%$ dos homens idosos tinham sobrepeso e/ou eram obesos ${ }^{31}$.

Em investigações longitudinais internacionais entre idosos com 60 anos e mais de idade, verificou-se, após followup de dois anos, que a não ocorrência de quedas foi mais frequente entre os homens ${ }^{13,34}$, contrapondo os achados da atual pesquisa. $O$ aumento percentual da não ocorrência de quedas entre as mulheres pode estar relacionado ao medo de cair, que em algumas situações, atua como um fator de proteção e, deste modo, evita que a idosa assuma um comportamento de risco durante suas atividades ${ }^{35}$. Destaca-se no atual estudo que apesar do menor percentual de mulheres que sofreram queda, observaram-se episódios recorrentes entre aquelas que caíram. Todavia, o receio de novas quedas pode reduzir a mobilidade e o condicionamento físico; e a capacidade de equilíbrio, e, consequentemente, resultar na reincidência de quedas ${ }^{12,35}$.

Identificou-se ainda aumento percentual de idosos do sexo masculino que caíram no follow-up, resultado que pode estar relacionado à piora do desempenho físico entre os homens na atual pesquisa. Em investigação longitudinal, com follow-up de dois anos, entre idosos de um município no interior de Minas Gerais verificou-se que o desempenho físico adequado foi um fator de proteção para quedas e episódios recorrentes ${ }^{12}$. Portanto, a identificação precoce das alterações relacionadas ao declínio do desempenho físico e a realização de intervenções adequada são essenciais para a prevenção desse agravo ${ }^{35}$.

Observou-se após o follow-up de quatro anos, diminuição no percentual de desempenho físico moderado/bom em ambos os sexos, sendo maior entre os homens. Tal resultado é semelhante às pesquisas longitudinais entre idosos norte-americanos ${ }^{15}$ e italianos ${ }^{36}$. Devido ao acúmulo de danos celulares e moleculares, que ocorrem durante o processo de envelhecimento humano e causam redução das reservas fisiológicas ${ }^{24}$, e, por conseguinte, declínio das habilidades físicas, mentais e psicossociais ${ }^{37}$, os idosos com idade mais avançada, geralmente, apresentam pior desempenho físico ${ }^{11,}$ 15,36. Essas alterações podem trazer consequências negativas para a população idosa, como a redução da mobilidade; isolamento social e agravos à saúde que demandam serviços especializados ${ }^{15,37}$. Tais fatores reforçam a necessidade de o desempenho físico ser avaliado durante a consulta de enfermagem gerontológica, visando trabalhar precocemente com os idosos e equipe multidisciplinar. O intuito é postergar os eventos adversos, considerando que o desempenho físico adequado auxilia na manutenção da capacidade funcional ${ }^{38}$, e com isso, o idoso é capaz de manter a independência e o autocuidado em saúde.

O aumento significativo na média do número de morbidade após o follow-up, em mulheres e homens idosos, se assemelha aos estudos longitudinais nacional ${ }^{10} \mathrm{e}$ internacional ${ }^{39}$ desenvolvidos na comunidade. Com o avançar da idade podem ocorrer alterações nos aspectos físicos, como o declínio da capacidade funcional e do desempenho físico ${ }^{14}$; questões psicossociais, dentre elas a baixa renda e escolaridade, acesso precário aos serviços de saúde, diminuição da rede e do apoio social ${ }^{39}$; e a adoção de hábitos de vida não saudáveis, a exemplo: alimentação inadequada, inatividade física, tabagismo e etilismo ${ }^{40}$. Tais fatores podem aumentar a vulnerabilidade dos idosos para desfechos adversos à saúde, como o maior número de morbidades ${ }^{14,39}$. Neste contexto, é essencial a adoção de estratégias para acolhimento e fortalecimento do vínculo profissional de saúde/idoso/familiares e a identificação precoce das morbidades visando à promoção da saúde e à prevenção de agravos. 
Ao longo do seguimento houve aumento do declínio funcional para a realização das AIVD em ambos os grupos, bem como verificado nos estudos longitudinais entre longevos na Colômbia ${ }^{16}$ e indivíduos com 60 anos ou mais de idade

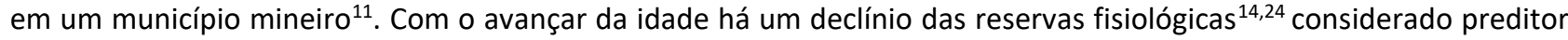
de desfechos adversos à saúde da população idosa, dentre eles a incapacidade funcional ${ }^{24}$. Os idosos com condições funcionais prejudicadas, que interferem na realização das AIVD, estão mais vulneráveis à ocorrência de quedas ${ }^{8}$, síndrome de fragilidade, violência e maus-tratos e à institucionalização ${ }^{38}$.

Entre as idosas, a média do número de quedas foi significativamente maior no follow-up, o que se assemelha às investigações nacional ${ }^{12}$ e internacional ${ }^{17}$. A ocorrência deste evento torna-se mais frequente com o avançar da idade, principalmente entre as mulheres, que além de sofrerem episódios recorrentes, estão mais susceptíveis a comorbidades, como a osteoporose, e à síndrome de fragilidade, consideradas fatores preditores de quedas ${ }^{17}$. Assim, o enfermeiro, por meio da consulta de enfermagem, visita domiciliária e atividades educativas, pode identificar os fatores de risco, que possam ocasionar episódios de quedas, possibilitando a proposição de medidas preventivas direcionadas aos idosos e familiares.

Também se verificou entre idosas suecas aumento significativo do número de componentes comprometidos do fenótipo de fragilidade no follow-up de dez anos ${ }^{17}$. Como observado em outros estudos, algumas condições de saúde e sociais presentes na maioria das mulheres desta investigação podem ter contribuído com esse resultado, como a presença de 5 ou mais morbidades ${ }^{3,14}$, redução da capacidade funcional para as AIVD ${ }^{24}$, quedas recorrentes ${ }^{17}$ e situação $^{2}$ econômica desfavorável ${ }^{2,26}$. Assim, o enfermeiro deve pautar seu processo de cuidado nas necessidades específicas dos idosos, considerando as diferenças entre os sexos, em busca da prevenção da síndrome de fragilidade e/ou minimização das suas consequências.

\section{Limitações do estudo}

A atual pesquisa tem como limitação o autorrelato das morbidades. Entretanto, a exclusão de idosos com declínio cognitivo minimiza o viés de seleção, ao evitar que aqueles com condições crônicas de saúde omitam esta informação. Ademais, os resultados fornecem subsídios para novas investigações, e sugere-se a realização de estudos multicêntricos e inquéritos nacionais, com amostras representativas da população idosa nos diversos estados brasileiros, a fim de contribuir com melhoria da atenção à saúde do idoso.

\section{CONCLUSÃO}

Ao longo do seguimento houve piora nas condições de saúde de mulheres e homens idosos, com 75 anos ou mais de idade, manifesto pelo aumento no número de morbidades e do declínio funcional para as AIVD. Especificamente, entre as idosas aumentaram, ainda, a ocorrência de quedas e piorou a condição de fragilidade.

Os achados podem contribuir com o direcionamento das intervenções de enfermagem voltadas à prevenção de agravos e à promoção da saúde das mulheres e homens idosos com idade avançada. Além disto, evidencia as diferenças nas condições de saúde entre os grupos, destacando as idosas, que possuem maior expectativa de vida e tornam-se mais vulneráveis às limitações funcionais e à pior qualidade de vida. Assim, reforça-se a necessidade de estratégias de intervenção de enfermagem específicas, uma vez que as mulheres e homens idosos podem apresentar padrões distintos de mudanças nas condições de saúde.

\section{REFERÊNCIAS}

1. Instituto Brasileiro de Geografia e Estatística. Projeção da população por sexo e idade - Indicadores implícitos na projeção 2020/2060. [Internet]. 2020 [cited 2020 Aug 18]. Available from: https://www.ibge.gov.br/apps/populacao/projecao/index.html.

2. Sousa NFS, Lima MG, Cesar CLG, Barros MBA. Active aging: prevalence and gender and age differences in a population-based study. Cad. Saúde Pública [Internet]. 2018 [cited 2021 Feb 5]; 34(11):e00173317. DOI: https://doi.org/10.1590/0102$311 \times 00173317$.

3. Grden CRB, Lenardt MH, Sousa JAV, Kusomota L, Dellaroza MSG, Betiolli SE. Associations between frailty syndrome and sociodemographic characteristics in long-lived individuals of a community. Rev. latinoam. Enferm. [Internet]. 2017 [cited 2021 Feb 5]; 25:e2886. DOI: https://doi.org/10.1590/1518-8345.1770.2886.

4. Queiroz DB, Araújo CM, Novais MM, Oliveira LC, Andrade LAA, Reis LA. Functionality, motor ability and health conditions in long-lived elderly people living at home. Arq. ciênc. Saúde [Internet]. 2016 [cited 2021 Feb 5]; 23(2):47-53. DOI: https://doi.org/10.17696/2318-3691.23.2.2016.281.

5. Pinto DS, Novais MM, Prates RV, Boas SV, Araujo CM, Reis LA. Functional activities and level of dependence in long-lived elderly living at home. Rev. Pesqui. Fisioter. [Internet]. 2017 [cited $2021 \mathrm{Feb} 5$ ]; 7(3):369-76. Available from: https://www5.bahiana.edu.br/index.php/fisioterapia/article/view/1500/943.

6. Krug RR, Schneider IJC, Giehl MWC, Antes DL, Confortin SC, Mazo GZ et al. Sociodemographic, behavioral, and health factors associated with positive self-perceived health of long-lived elderly residents in Florianópolis, Santa Catarina, Brazil. Rev. bras. epidemiol. [Internet]. 2018 [cited 2021 Feb 5]; 21:e180004. DOI: https://doi.org/10.1590/1980-549720180004. 
7. Trevisan C, Veronese N, Maggi S, Baggio G, Toffanello ED, Zambon S et al. Factors influencing transitions between frailty states in elderly adults: the progetto Veneto Anziani longitudinal study. J. am. geriatr. [Internet]. 2016 [cited 2021 Feb 5]; 65(1):17984. DOI: https://doi.org/10.1111/jgs.14515.

8. Carneiro JA, Lima CA, Costa FM, Caldeira AP. Health care are associated with worsening of frailty in community older adults. Rev. Saúde Pública [Internet]. 2019 [cited 2021 Feb 5]; 53(1):32. DOI: https://doi.org/10.11606/s1518-8787.2019053000829.

9. Duarte YAO, Nunes DP, Andrade FB, Corona LP, Brito TRP, Santos JLF. Frailty in older adults in the city of São Paulo: Prevalence and associated factors. Rev. bras. epidemiol. [Internet]. 2018 [cited 2021 Feb 5]; 21(Suppl 2):e180021. DOI: https://doi.org/10.1590/1980-549720180021.supl.2.

10. Confortin SC, Schneider IJC, Antes DL, Cembranel F, Ono LM, Marques LP et al. Life and health conditions among elderly: results of the EpiFloripa Idoso cohort study. Epidemiol. Serv. Saúde [Internet]. 2017 [cited 2021 Feb 5]; 26(2):305-17. DOI: https://doi.org/10.5123/s1679-49742017000200008.

11. Ikegami EM, Souza LA, Tavares DMS, Rodrigues LR. Functional capacity and physical performance of community-dwelling elderly: a longitudinal study. Ciênc. saúde colet. [Internet]. 2020 [cited 2021 Feb 5]; 25(3):1083-90. DOI: https://doi.org/10.1590/1413-81232020253.18512018.

12. Souza AQ, Pegorari MS, Nascimento JS, Oliveira PB, Tavares DMS. Incidence and predictive factors of falls in communitydwelling elderly: a longitudinal study. Ciênc. saúde colet. [Internet]. 2019 [cited 2021 Feb 5]; 24(9):3507-16. DOI: https://doi.org/10.1590/1413-81232018249.30512017.

13. Wu H, Ouyang P. Fall prevalence, time trend and its related risk factors among elderly people in China. Arch. gerontol. geriatr. [Internet]. 2017 [cited 2021 Feb 5]; 73(1):294-9. DOI: https://doi.org/10.1016/j.archger.2017.08.009.

14. Fhon JRS, Rodrigues RAP, Santos JLF, Diniz MA, Santos EB, Almeida VC. Factors associated with frailty in older adults: a longitudinal study. Rev. Saúde Pública [Internet]. 2018 [cited 2021 Feb 5]; 52:74. DOI: https://doi.org/10.11606/s15188787.2018052000497.

15. Botoseneanu A, Allore HG, Leon CFM, Gahbauer EA, Gill TM. Sex Differences in Concomitant Trajectories of Self-Reported Disability and Measured Physical Capacity in Older Adults. J Gerontol A Biol Sci Med Sci. [Internet]. 2016 [cited 2021 Feb 5]; 71(8):1056-62. DOI: https://doi.org/10.1093/gerona/glw038.

16. Tindale LC, Salema D, Brooks-Wilson AR. 10-year follow-up of the Super-Seniors Study: compression of morbidity and genetic factors. BMC geriatr. [Internet]. 2019 [cited 2021 Feb 5]; 58(19):1-7. DOI: https://doi.org/10.1186/s12877-019-1080-8.

17. Bartosch PS, Kristensson J, McGuigan FE, Akesson KE. Frailty and prediction of recurrent falls over 10 years in a community cohort of 75-year-old women. Aging Clin Exp Res. [Internet]. 2020 [cited 2021 Feb 5]; 1-10. DOI: https://doi.org/10.1007/s40520-019-01467-1.

18. Bertolucci PHF, Brucki SMD, Campacci SR, Juliano Y. The mini-mental state examination in an outpatient population: influence of literacy. Arq. Neuro-Psiquiatr. [Internet]. 1994 [cited 2021 Feb 5]; 52(1):1-7. DOI: https://doi.org/10.1590/S0004282X1994000100001.

19. Santos RL, Virtuoso Junior JS. Reliability of the Brazilian version of the Scale of Instrumental Activities of Daily Living. Rev. Pesqui. Fisioter. [Internet]. 2008 [cited 2020 Aug 27]; 21(4):290-6. Available from: https://periodicos.unifor.br/RBPS/article/viewFile/575/2239.

20. Fried LP, Tangen CM, Walston J, Newman AB, Hirsch C, Gottdiener J et al. Cardiovascular Health Study Collaborative Research Group. Frailty in older adults evidence for a phenotype. J Gerontol A Biol Sci Med Sci. [Internet]. 2001 [cited 2021 Feb 5]; 56(3):146-57. DOI: https://doi.org/10.1093/gerona/56.3.m146.

21. Tavares DMS, Faria PM, Pegorari MS, Ferreira PCS, Nascimento JS, Marchiori, GF. Frailty Syndrome in Association with Depressive Symptoms and Functional Disability among Hospitalized Elderly. Issues Ment Health Nurs. [Internet]. 2018 [cited 2021 Feb 5]; 39(5):433-8. DOI: https://doi.org/10.1080/01612840.2018.1429035.

22. World Health Organization (WHO). Physical status: the use and interpretation of antropometry. Report of a WHO Expert Commitee. WHO Technical Report Series, 854. [Internet]. Geneve: WHO; 1995 [cited 2020 Aug 11]. Available from: https://www.who.int/childgrowth/publications/physical_status/en/.

23. Nakano MM. Versão brasileira da Short Physical Performance Battery SPPB: adaptação cultural e estudo da confiabilidade [dissertação]. Campinas (SP): Faculdade de Educação, Universidade Estadual de Campinas; 2007. 163 p. Available from: http://repositorio.unicamp.br/jspui/handle/REPOSIP/252485.

24. Fernandes DS, Gonçalves LHT, Ferreira AMR, Santos MIPO. Functional capacity assessment of long-lived older adults from Amazonas. Rev. bras. enferm. [Internet]. 2019 [cited 2021 Feb 5]; 72(Supl. 2):49-55. DOI: https://doi.org/10.1590/0034-71672017-0798.

25. Riani JLR, Marinho KRL, Ferreira FPM, Camargos MCS. Labor market and income differentials of the Minas Gerais State elderly population. Pretexto [Internet]. 2018 [cited 2021 Feb 5]; 19(4):11-29. DOI: http://dx.doi.org/10.21714/pretexto.v19i4.5377.

26. Carmel S. Health and Well-Being in Late Life: Gender Differences Worldwide. Front. Med. [Internet]. 2019 [cited 2021 Feb 5]; 56(2):113-31. DOI: https://dx.doi.org/10.3389\%2Ffmed.2019.00218.

27. Nunes DP, Brito TRP, Giacomin KC, Duarte YAO, Lebrão ML. Performance pattern of activities of daily living for older adults in the city of São Paulo in 2000, 2006, and 2010. Rev. bras. epidemiol. [Internet]. 2018 [cited 2021 Feb 5]; 21(Supl 2):e180019. DOI: https://doi.org/10.1590/1980-549720180019.supl.2.

28. Bortoluzzi EC, Doring M, Portella M, Cavalcanti G, Mascarelo A, Dellani M. Prevalence and factors associated with functional dependence in the oldest old. Rev. Bras. Ativ. Fís. Saúde [Internet]. 2017 [cited 2021 Feb 5]; 22(1):85-94. DOI: https://doi.org/10.12820/rbafs.v.22n1p85-94. 
29. Boerma T, Hosseinpoor AR, Verdes E, Chatterji S. A global assessment of the gender gap in self-reported health with survey data from 59 countries. BMC Public Health [Internet]. 2016 [cited 2021 Feb 5];16(1):675. DOI: https://doi.org/10.1186/s12889016-3352-y.

30. Lins MEM, Marques APO, Leal MCC, Barros RLM. Frailty risk in community-dwelling elderly assisted in Primary Health Care and associated factors. Saúde Debate [Internet]. 2019 [cited 2021 Feb 5]; 43(121):520-9. DOI: https://doi.org/10.1590/01031104201912118

31. Kim KH, Park SK, Lee DR, Lee J. The Relationship between Handgrip Strength and Cognitive Function in Elderly Koreans over 8 Years: A Prospective Population-Based Study Using Korean Longitudinal Study of Ageing. Korean J Fam Med. [Internet]. 2019 [cited 2021 Feb 5]; 40(1):9-15. DOI: https://doi.org/10.4082/kjfm.17.0074.

32. Landi F, Calvani R, Tosato M, Martone AM, Ortolani E, Savera G et al. Anorexia of Aging: Risk Factors, Consequences, an Potential Treatments. Nutrients [Internet]. 2016 [cited 2021 Feb 5]; 8(2):1-10. DOI: https://doi.org/10.3390/nu8020069.

33. Liberalesso TEM, Dallazen F, Bandeira VAS, Berlezi EM. Prevalence of frailty in a long-lived population in the Southern region of Brazil. Saúde Debate [Internet]. 2017 [cited 2021 Feb 5]; 41(113):553-62. DOI: https://doi.org/10.1590/0103-1104201711316.

34. Gale CR, Cooper C, Aihie AS. Prevalence of falls and associated factors in elderly individuals. Age and Ageing [Internet]. 2016 [cited 2021 Feb 5]; 45(6):789-94. Available from: https://www.scielosp.org/article/csp/2019.v35n8/e00115718/.

35. Vitorino LM, Teixeira CAB, Boas ELV, Pereira RL, Santos NO, Rozendo CA. Fear of falling in older adults living at home: associated factors. Rev. esc. enferm. USP [Internet]. 2017 [cited 2021 Feb 5]; 51:e03215. DOI: https://doi.org/10.1590/s1980$220 \times 2016223703215$.

36. Martinez-Gomez D, Bandinelli S, Del-Panta V, Patel KV, Guralnick JM, FerruCCi L. Three-Year Changes in Physical Activity and Decline in Physical Performance Over 9 Years of Follow-Up in Older Adults: The Invecchiare in Chianti Study. J Am Geriatr Soc. [Internet]. 2017 [cited 2021 Feb 5]; 65(6):1176-82. DOI: https://doi.org/10.1111/jgs.14788.

37. Organização Mundial da Saúde (OMS). Relatório mundial de envelhecimento e saúde. [Internet]. 2015 [cited 2020 Aug 11 ]. Available from: http://sbgg.org.br/wp-content/uploads/2015/10/OMS-ENVELHECIMENTO-2015-port.pdf.

38. Araújo GKN, Souto RQ, Alves FAP, Sousa RCR, Ceballos AGC, Santos RC et al. Functional capability and associated factors in living in a community. Acta Paul. Enferm. [Internet]. 2019 [cited 2021 Feb 5]; 32(3):312-8. DOI: https://doi.org/10.1590/19820194201900043.

39. Singer L, Green M, Rowe F, Ben-Shlomo Y, Morrissey K. Social determinants of multimorbidity and multiple functional limitations among the ageing population of England, 2002-2015. SSM - Population Health [Internet]. 2019 [cited 2021 Feb 5] 8(1):e100413. DOI: https://doi.org/10.1016/j.ssmph.2019.100413. 